\title{
Imaging Attentional Modulation of Pain in the Periaqueductal Gray in Humans
}

\author{
Irene Tracey, ${ }^{1}$ Alexander Ploghaus, ${ }^{1}$ Joseph S. Gati, ${ }^{2}$ Stuart Clare, ${ }^{1}$ Steve Smith, ${ }^{1}$ Ravi S. Menon, ${ }^{2}$ and \\ Paul M. Matthews 1 \\ ${ }^{1}$ Centre for Functional Magnetic Resonance Imaging of the Brain, Department of Clinical Neurology, University of Oxford, \\ Oxford OX3 9DU, United Kingdom, and 'Laboratory for Functional Magnetic Resonance Research, John P. Robarts \\ Research Institute, London, Ontario N6A 5K8, Canada
}

Pain is an unpleasant sensory and emotional experience usually triggered by stimulation of peripheral nerves and often associated with actual or potential tissue damage. It is well known that pain perception for patients and normal subjects can be modulated by psychological factors, such as attention, stress, and arousal. Our understanding of how this modulation occurs at a neuroanatomical level is poor. Here we neuroanatomically defined a key area in the network of brain regions active in response to pain that is modulated by attention to the painful stimulus. High-resolution functional magnetic resonance imaging was used to define brain activation to painful heat stimulation applied to the hand of nine normal subjects within the periaqueductal gray region. Subjects were asked to either focus on or distract themselves from the painful stimuli, which were cued using colored lights. During the distraction condition, subjects rated the pain intensity as significantly lower compared with when they attended to the stimulus. Activation in the periaqueductal gray was significantly increased during the distraction condition, and the total increase in activation was predictive of changes in perceived intensity. This provides direct evidence supporting the notion that the periaqueductal gray is a site for higher cortical control of pain modulation in humans.

Key words: pain; fMRI; PAG; attention; modulation; brainstem
Control of pain in both acute and chronic conditions is a major medical problem. Although much pain is a consequence of stimulation of peripheral nociceptors, the CNS plays a major role in the processing of all noxious sensations (Wall and Melzack, 1999). Activation of a specific network of brain regions is associated with the perception of pain, and candidate brain regions processing sensory-discriminative and emotional-affective components of pain perception are being defined (Albe-Fessard et al., 1985; Rainville et al., 1997; Ploghaus et al., 1999, 2000; Ploner et al., 1999; Petrovic et al., 2000; Tracey et al., 2000).

Under certain conditions, however, it is possible to block the perception of pain despite noxious stimulation. A lack of reported pain by soldiers during battle, despite severe injuries, and experimental observations of changes in responses to painful stimuli with changes in psychological factors, such as arousal, attention, stress, and mood state, demonstrate that pain perception varies with context (Melzack et al., 1982; Gaughan and Gracely, 1989). That the relationship between reported pain and stimulus intensity is highly variable led Head and Holmes (1911) to postulate that the psychological state of the subject can modulate the perception of pain. One critical element of the psychological state that can powerfully modulate subjective responses to noxious stimuli is attention. Decreased attention to noxious stimuli raises the pain threshold (Miron et al., 1989), whereas perceived pain intensity is increased when a subject's attention is directed to

\footnotetext{
Received May 7, 2001; revised Jan. 11, 2002; accepted Jan. 15, 2002.

This work was supported by the Medical Research Council (UK) (I.T., S.S., and P.M.M.). We thank Dermot Dobson for technical support.

Correspondence should be addressed to Dr. Irene Tracey, Centre for Functional Magnetic Resonance Imaging of the Brain, John Radcliffe Hospital, Headington, Oxford OX3 9DU, UK. E-mail: irene@fmrib.ox.ac.uk.

Copyright (C) 2002 Society for Neuroscience $0270-6474 / 02 / 222748-05 \$ 15.00 / 0$
}

painful stimuli (Bushnell et al., 1985). Indeed, distraction is used as an adjunct in pain management (Good et al., 1999).

There is limited information, however, concerning the central mechanisms by which attentional changes modulate pain perception (Fields and Basbaum, 1999; Petrovic et al., 2000). One potential site for mediation of modulatory impulses from higher centers was suggested with the description of stimulationproduced analgesia (SPA) (Reynolds, 1969; Mayer et al., 1971; Mayer and Price, 1976). SPA is produced by electrical stimulation of discrete brain sites and produces inhibition of reflex responses to noxious stimulation, such as tail flick in rats. An SPA effect is also generated in humans by direct stimulation of the periaquectual gray (PAG) (Boivie and Meyerson, 1982; Baskin et al., 1986). The PAG may therefore be a critical center for control of peripheral nociceptive perception by descending activity from higher cortical regions.

Here we describe functional magnetic resonance imaging (fMRI) studies that test directly whether the PAG is part of a descending pathway for attentional control of pain. Specifically, we tested whether activation changes in the PAG accompany changes in attention to a painful stimulus. To optimize the sensitivity for detection of PAG activation by fMRI, we collected functional imaging data using a high-field (4 tesla) imaging system to enhance the relative blood oxygen level-dependent (BOLD) contrast contribution from the parenchyma rather than any draining veins (Gati et al., 1997). In addition, we make use of the greater signal-to-noise available at high field to acquire images at a higher spatial resolution than used typically for fMRI studies and restricted our data collection to slices within the PAG region.

\section{MATERIALS AND METHODS}

Subjects. Nine right-handed volunteers were studied (six males, three females; mean \pm SD age, $26 \pm 2.6$ years). All subjects gave informed 
consent, and the study was approved both by the Oxford Committee for Research Ethics and the University of Western Ontario Ethics Review Board. Noxious and warm thermal stimuli were applied to the dorsum of the right hand with a $3 \times 3 \mathrm{~cm}$ Peltier thermode, designed and built in-house. In the scanner, an adaptive procedure was used to identify two stimuli consistently described for each subject as "painfully hot, moderate-strong pain" and "clearly warm, but not painful." Subjects were instructed which of two of three possible light-emitting diodes (LEDs), observable from the magnet bore (red, blue, or green), would signify onset of either painful heat or nonpainful warm stimulation, with no delay between LED and stimulus onset. The LEDs would remain on throughout the duration of stimulation. Each subject received five bursts of painful heat and five bursts of nonpainful warm stimulation, in which stimulus duration lasted $12 \mathrm{sec}$, in a randomized manner. The assignment of LED color to intensity of stimulation was randomized across subjects. Subjects underwent two experiments within the same imaging session. In one session, they were instructed to pay full attention to the painful or warm stimulation (denoted "attending" or "A" task) and, in the second, to try and decrease the perception of painful or warm stimulation by not attending (i.e., think of something else) to the stimulus (denoted "not attending" or "NA" task). All subjects understood the instructions and cooperated, based on postexperimental interviews. The order of the two tasks was randomized across subjects. After the imaging data were completed and subjects were removed from the scanner, they rated both the intensity and unpleasantness of thermal stimulation in both conditions using an 11 point scale with verbal descriptors. On the basis of previous work, we expected subjects to rate the NA pain condition lower than the A pain condition; ratings between conditions were analyzed for significance using a one-tailed Student's $t$ test.

Imaging. Data were acquired on a 4 tesla whole-body imaging system (Varian, Palo Alto, CA; Siemens, Erlangen, Germany) with a hybrid birdcage transmit-receive radio frequency coil. The subject's head was placed into a head holder and packed with foam to reduce motion. T1-weighted sagittal scout images were used to select seven contiguous 4 $\mathrm{mm}$ axial slices (obliquely oriented) through the mesencephalon. Each functional volume was acquired using a navigator echo-corrected, interleaved multishot (four shots) echo planar imaging pulse sequence with a $128 \times 128$ matrix size and a total volume acquisition time of $2.5 \mathrm{sec}$ [echo time (TE), $15 \mathrm{msec}$; flip angle, $50^{\circ}$; field of view (FOV), $22.0 \mathrm{~cm}$ ]. During each imaging session, high-resolution $(256 \times 256)$ three-dimensional T1-weighted structural volumes were acquired in the same FOV and orientation as the functional images (TE, $6 \mathrm{msec}$; repetition time, 11 msec; inversion time, $500 \mathrm{msec}$; flip angle, $11^{\circ}$ ). The resulting acquisition produced 64 contiguous structural images, each with a slice thickness of $1.0 \mathrm{~mm}$.

Image processing and quantification. Image processing and statistical analyses were performed with MEDx (Sensor Systems, Sterling, VA). All volumes were realigned and smoothed with a $3.0 \times 3.0 \times 3.0 \mathrm{~mm}^{3}$ (full-width at half-maximum) Gaussian kernel, and the average signal intensity of every volume was normalized to the same mean value. Temporal filtering was applied (high-pass cutoff of $200 \mathrm{sec}$ ), and activation maps were calculated (for illustrative purposes only) by parametric unpaired $t$ tests between volumes collected during noxious heat stimulation and those collected during non-noxious warm stimulation (denoted pain minus warm or " $\mathrm{P}-\mathrm{W}$ ").

Mean difference images were generated by subtracting BOLD signal time course data obtained during non-noxious warm stimulation from the BOLD signal time course data obtained during noxious heat stimulation. These mean difference images were resliced into high-resolution space so that a manually defined mask of the periaqueductal gray, defined by the tissue borders seen on the high-resolution anatomical images, could be overlaid onto the mean difference images. The mean signal intensity difference for each voxel within the PAG between noxious heat and non-noxious warm stimulation for each subject was determined under both conditions using this mask [denoted $(\mathrm{P}-\mathrm{W})+\mathrm{A}$ for the attending to pain relative to warm condition and $(\mathrm{P}-\mathrm{W})+\mathrm{NA}$ for the not attending to pain relative to warm condition]. A Student's $t$ test (matched, one-tailed) of these results was performed between the $(\mathrm{P}-\mathrm{W})+\mathrm{A}$ and $(\mathrm{P}-\mathrm{W})+\mathrm{NA}$ conditions to test whether a significant increase in activation occurred during the $(\mathrm{P}-\mathrm{W})+\mathrm{NA}$ condition. This was to determine whether a larger perceptual decrease in pain intensity or aversiveness correlated with a larger total activity within the PAG. This method of analysis is a more objective measure of the total activity within the PAG compared with a threshold-based activity summary (whether calculated from $Z$ statistic or activation level images). The latter depends more subjectively
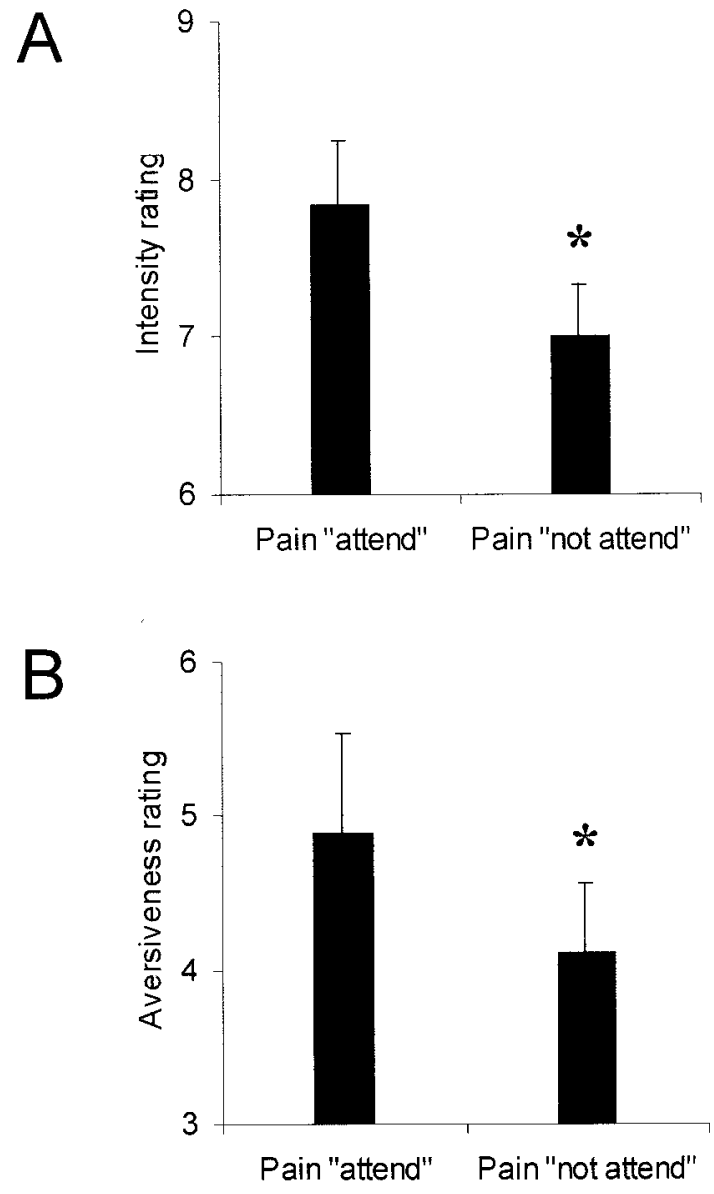

Figure 1. $A, B$, Graphs showing the pain scores (mean $\pm \mathrm{SE}$ ) between conditions for intensity $(A)$ and aversiveness $(B)\left({ }^{*} p<0.05\right)$.

on factors of little relative interest (such as noise levels) and arbitrary objectivity (such as actual thresholds used). In addition, a correlation analysis was done between the total change in visual analog score (VAS) rating (intensity and aversiveness) and total change in activity within the PAG for the two conditions.

\section{RESULTS}

\section{Behavioral responses to stimuli}

Figure 1, $A$ and $B$, displays the pain scores for intensity and aversiveness during the two attentional conditions [perceived pain while not attending to pain $(\mathrm{P}+\mathrm{NA})$ and perceived pain while attending to pain $(\mathrm{P}+\mathrm{A})]$. A significant decrease in the rating for pain was found during the $\mathrm{P}+\mathrm{NA}$ condition compared with the $\mathrm{P}+\mathrm{A}$ condition (mean $\pm \mathrm{SE} ; \mathrm{P}+\mathrm{A}, 7.8 \pm 0.4$ vs $\mathrm{P}+\mathrm{NA}$, $7.0 \pm 0.3 ; t=3.51 ; p=0.004$ ) was found. Aversiveness (VAS) of the stimuli was also rated to be lower during the $\mathrm{P}+\mathrm{NA}$ condition compared with the $\mathrm{P}+\mathrm{A}$ condition (mean $\pm \mathrm{SE} ; \mathrm{P}+\mathrm{A}, 4.9 \pm 0.7$ vs $\mathrm{P}+\mathrm{NA}, 4.1 \pm 0.4 ; t=2.15 ; p=0.03)$. We did not find any significant differences in the ratings of warm stimulation during the two conditions (intensity ratings: mean $\pm \mathrm{SE}$; warm $+\mathrm{A}$, $2.78 \pm 0.32$ vs warm $+\mathrm{NA}, 2.56 \pm 0.38 ; p=0.26$ ) (aversiveness ratings: mean $\pm \mathrm{SE}$; warm $+\mathrm{A}, 1.17 \pm 0.17$ vs warm $+\mathrm{NA}, 0.94 \pm$ 0.18; $p=0.09)$.

\section{fMRI measurements of the periaqueductal gray response to noxious stimulation}

Using high-resolution echo planar imaging at 4 tesla, activation within the PAG in response to noxious thermal stimulation can be 


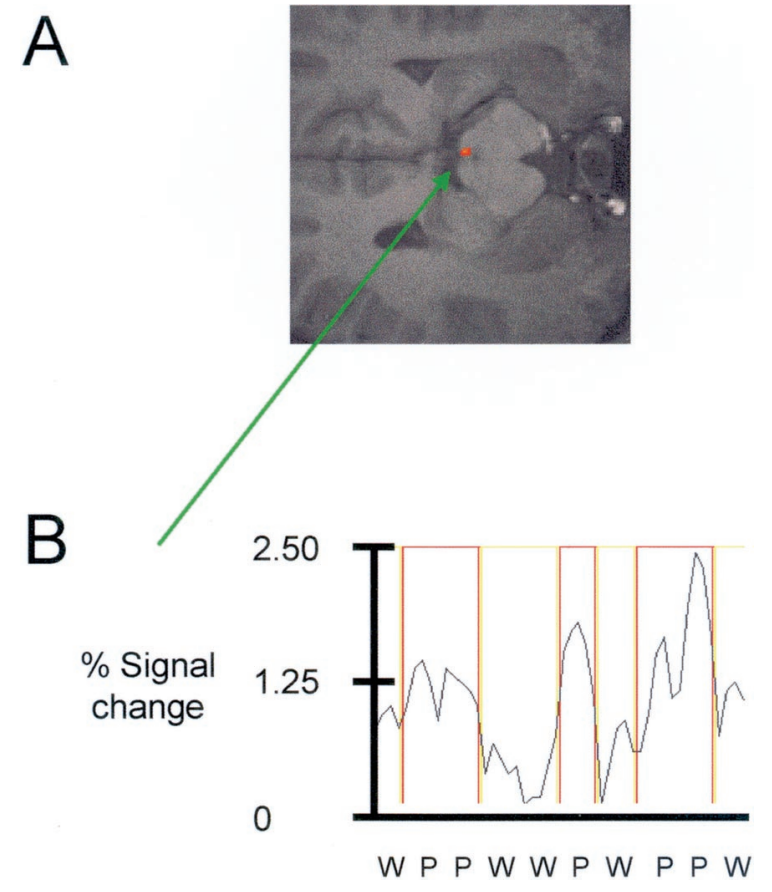

Figure 2. $A, B$, Figure showing representative activation within the periaqueductal gray for one subject $(A)$. The corresponding time course of MR signal intensity change during warm (yellow boxes) and painful (red boxes) stimulation for the voxel with highest $Z$ statistic is also shown $(B)$.

demonstrated even for individual subjects (Fig. $2 A$ ). A representative time course for the single voxel with highest $Z$ statistic in the activated region is displayed in Figure $2 B$. This time course is derived from concatenated data taken from the periods when subjects received a warm (marked in yellow outline) or a painful hot (marked in red outline) stimulus. There was increased signal intensity $(\sim 0.6-1.8 \%)$ in the PAG region during noxious stimulation relative to periods of pleasant warmth. We did not find any significant activation of the PAG region during warm stimulation alone (i.e., during warm minus no stimulation; results not shown).

To confirm that PAG activation was enhanced for the pain activation in the not attend condition $[(\mathrm{P}-\mathrm{W})+\mathrm{NA}]$ condition compared with the pain activation in the attend condition $[(\mathrm{P}-\mathrm{W})+\mathrm{A}]$ condition, we adopted a region-of-interest analysis of the time course data from the $\mathrm{P}-\mathrm{W}$ comparison for each subject. The region-of-interest (defined from a high-resolution structural scan) was limited to the PAG. For each voxel within the $\mathrm{PAG}$, the mean difference of $\mathrm{P}-\mathrm{W}$ was obtained on a voxel-byvoxel basis (see Materials and Methods). These values were summed for each subject. The contrast of NA-A showed a mean $25 \%$ signal increase [mean \pm SE (arbitrary units); NA, $1981 \pm$ 384 vs $\mathrm{A}, 1487 \pm 278]$ in the total signal intensity within the PAG $(p=0.02)$ (Fig. 3A). There was a significant correlation $(p<$ 0.025 ) (Fig. $3 B$ ) between the total activity change in the PAG between conditions and the total change in VAS for intensity, i.e., a greater change in PAG activity is found when a larger decrease in pain intensity is reported. There was no significant correlation with the aversiveness ratings. It is clear from Figure 3, $A$ and $B$, that there are reasonable differences in the amount of activation to painful stimulation within the PAG across subjects; nevertheless, these are not so great that significant differences in the means and correlation to subjective behavioral data could not be detected.

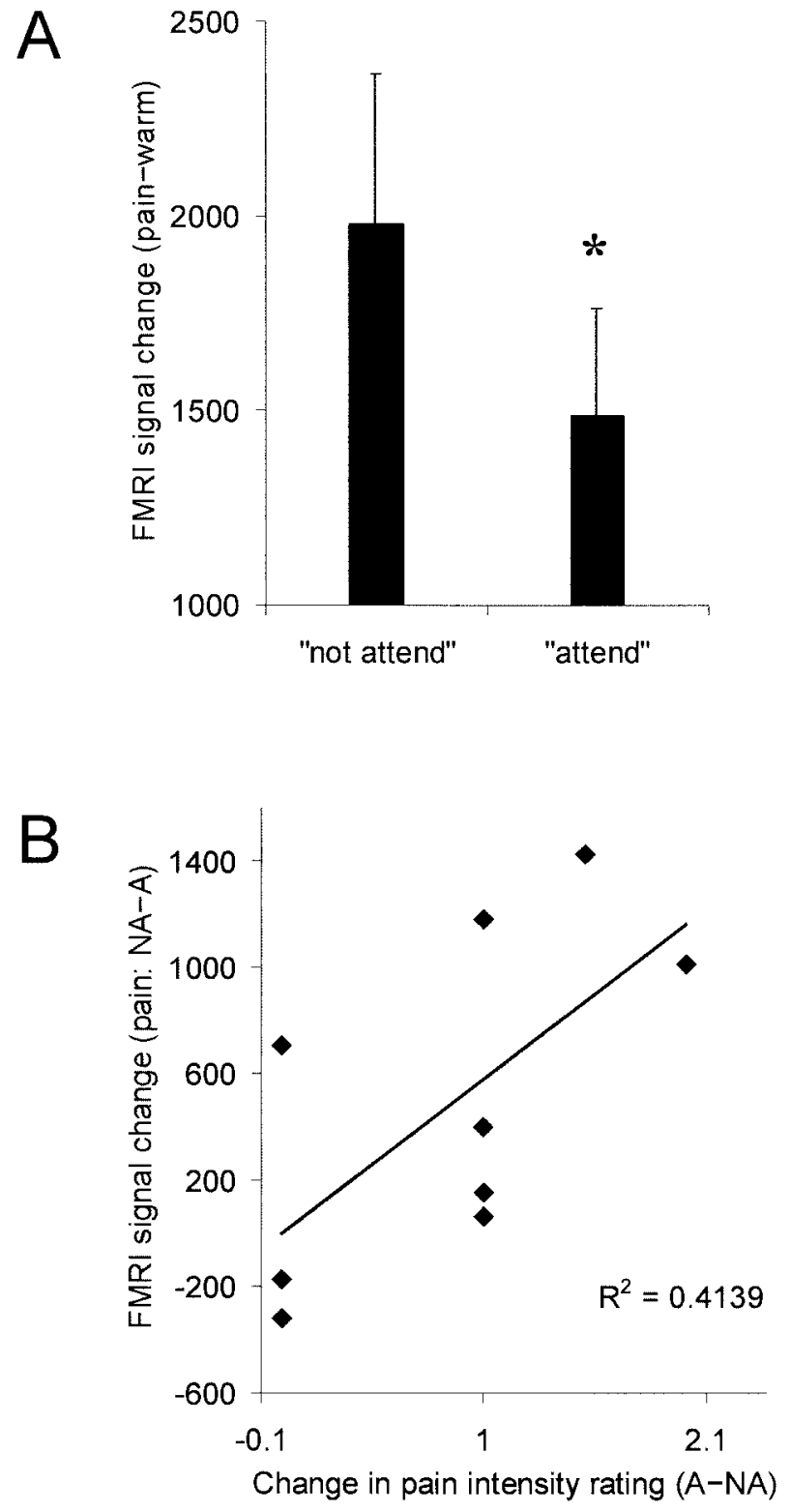

Figure 3. $A, B$, Total signal intensity (arbitrary units) within the periaqueductal gray for the two attentional conditions $(A)$ (mean $\pm \mathrm{SE} ;{ }^{*} p<$ $0.05)$. $B$, Correlation of total signal intensity change (arbitrary units) within the periaqueductal gray and total change in pain intensity (visual analog score) between the two conditions $(p<0.025)$.

\section{DISCUSSION}

Consistent with previous behavioral studies (Bushnell et al., 1985; Miron et al., 1989; Fields and Basbaum, 1999), we found a significant decrease in the behavioral ratings of pain for both intensity and aversiveness in the $\mathrm{P}+\mathrm{NA}$ condition compared with the $\mathrm{P}+\mathrm{A}$ condition (Fig. $1 A, B$ ). This study was designed to test whether the PAG is one candidate brain structure for this "topdown" influence on pain perception.

Our results have demonstrated first that high-field fMRI can be used to monitor pain-specific activation within the PAG (Fig. $2 A, B)$. Second, we showed a significant increase in activation associated with our noxious thermal stimulus specifically within the PAG when subjects were distracted from the pain (Fig. 3A). That this difference was related directly to the perception of pain 
was emphasized by the observation that the activation difference was significantly correlated with the total VAS (intensity) change between conditions (Fig. 3B). That is the total signal within the PAG is greater when the difference in perceptual rating of pain intensity for the two conditions is higher (Fig. $3 B$ ). In conjunction with previous studies showing that direct electrical stimulation of the PAG induces analgesia (Reynolds, 1969; Akil et al., 1976; Boivie and Meyerson, 1982; Baskin et al., 1986), these results support the hypothesis that increased activity within the PAG correlates with perceptual decreases in pain intensity. It should be noted, however, that because of technical limitations, we were not able to record task performance for reliable distraction or subjective pain and warm ratings during the experiment itself. Subjective ratings of thermal stimulation were taken during postexperimental interviews. This might therefore introduce memory bias, and future studies would benefit from concurrent ratings of pain perception. In addition, future studies should obtain behavioral measures of attention or distraction to confirm reliable task performance. However, we believe our results that show a significant correlation between the behavioral and imaging data support the conclusion that subjects reported pain ratings reliably and that neither memory bias nor task performance for reliable distraction were major confounds in this study.

There is a large body of evidence to date suggesting that changes in pain responses attributable to changes in arousal or attention result from the action of modulatory networks that control the transmission of nociceptive signals to the brain (Hagbarth and Kerr, 1954; Duncan et al., 1987; Oliveras et al., 1990; Petrovic et al., 2000). This evidence is mostly based on animal studies, because methods to investigate such modulatory networks in humans have not been available until recently. One site for modulation of the pain response is the medullary dorsal horn (trigeminal nucleus caudalis). Electrophysiological studies have demonstrated that activity of the trigeminal nucleus caudalis is increased with anticipation of a noxious stimulus (a process undoubtedly mediated by cortical centers) in a similar way to increases with pain itself (Duncan et al., 1987). Another site proposed, and one of the first regions suggested as being responsible for pain modulation, was the PAG (Reynolds, 1969; Mayer et al., 1971; Mayer and Price, 1976). However, it is not known whether cortical inputs to the PAG are a route by which such cognitive inputs can exert their influence on sensory afferent input in humans.

Neuroanatomical evidence suggests that the PAG could mediate central modulation of ascending sensory responses. The PAG receives major inputs from the frontal cortex, hypothalamus (Beitz, 1982), frontal granular, insular cortex (Hardy and Leichnetz, 1981), and amygdala (Gray and Magnuson, 1992). In addition, there are several major brainstem inputs to the PAG (Basbaum and Fields, 1984; Herbert and Saper, 1992). The caudal PAG projects to the rostral ventromedial medulla (RVM), which in turn sends projections to pain-transmitting neurons in the dorsal horn of the spinal cord and the trigeminal nucleus caudalis. Just like stimulation of the PAG, electrical stimulation of the RVM produces analgesia and inhibits dorsal horn pain transmission neurons (Akil et al., 1976; Baskin et al., 1986).

Pain-modulating neurons in the PAG and RVM have very large, virtually total body "receptive fields" that project diff usely to multiple levels of the neuroaxis, including the trigeminal nucleus, the dorsal horn at multiple spinal levels, and RVM neurons. Cells throughout this network can fire at the same time, suggesting that the PAG-RVM network functions as a unit and exerts global rather than topographically discrete control over dorsal horn pain transmission neurons. Such an arrangement is therefore consistent with this system being integrated with behavioral functions of arousal and attention. It is therefore possible that the RVM is also critical for attentional modulation of descending mechanisms. We were not able to test this directly with the present experiment, because to obtain adequate spatial resolution to determine PAG activation, we were limited in the number of slices that could be taken per volume. This prevented us from prescribing slices as far down as the medulla. Additional work is needed to characterize these other regions and their role in mediating top-down pain modulation.

One mechanism by which the PAG modulates pain perception involves the release of endogenous opioids. The PAG contains significant quantities of all families of endogenous opioid peptides, and it is known that $\mu$ opioids act by releasing PAG projection cells from GABAergic inhibition (Fields and Basbaum, 1999). Stress-induced analgesia in animals and placebo analgesia in humans with postoperative pain are reduced by the opioid antagonist naloxone (Akil et al., 1976; Watkins and Mayer, 1982; Watkins et al., 1982). It is possible, therefore, that attentional mechanisms act by releasing endogenous opioids within the PAG.

One possible model for our findings is that, during distraction, there is increasing activation of the PAG to enable release of endogenous opioids that exert their antinociceptive effect through the opioid synapses of the RVM system. In the PAG to RVM system, opiates inhibit RVM "off” cells (Fields and Basbaum, 1999). Disinhibition of off cells enables them to exert their antinociceptive effect on dorsal horn pain transmission neurons. A test of this hypothesis would be to administer the opiate antagonist naloxone, which should abolish the attentional modulation of perceived pain intensity but not the increased PAG activation with distraction. A study by Moret et al. (1991), however, suggests that one form of cognitive analgesia (hypnotic analgesia) was not reversed by naloxone, suggesting that the opiate endorphin system might not primarily mediate all forms of cognitive analgesia. Future work will investigate specifically how higher cognitive brain structures during distraction tasks activate the PAG.

There are precedents for central attentional modulation of brainstem nuclei by attentional tasks. For example, McGlone found that fMRI activation in the human superior colliculus was modulated during a tactile attention task (McGlone et al., 1999). Lavernhe-Lemaire and Robier (1997) had subjects alternately relax or focus their attention on auditory input from one side only during simultaneous recording of brainstem-evoked potentials. The brainstem wave III amplitude was decreased on the side where attention was focused, providing evidence that, when attention is focused, as done in our study, one can lower brainstemevoked potentials. This supports the notion that distraction from sensory perception may reduce inhibition of specific brainstem centers.

To our knowledge, the present study therefore is the first imaging study to show that cognitive processes can modulate pain-associated activity specifically within the PAG. Our method provides an in vivo assay for objectively monitoring pain modulation in humans and provides evidence that the PAG is a route for top-down influences on the descending pain inhibitory pathways in humans.

\section{REFERENCES}

Akil H, Mayer DJ, Liebeskind JC (1976) Antagonism of stimulationproduced analgesia by naloxone, a narcotic antagonist. Science
191:961-962. 
Albe-Fessard D, Berkley KJ, Kruger L, Ralston III HJ, Willis Jr WD (1985) Diencephalic mechanisms of pain sensation. Brain Res 3:217-296.

Basbaum AI, Fields HL (1984) Endogenous pain control systems: brainstem spinal pathways and endorphin circuitry. Annu Rev Neurosci 7:309-338.

Baskin DS, Mehler WR, Hosobuchi Y, Richardson DE, Adams JE, Flitter MA (1986) Autopsy analysis of the safety, efficacy and cartography of electrical stimulation of the central gray in humans. Brain Res 371:231-236.

Beitz AJ (1982) The sites of origin brain stem neurotensin and serotonin projections to the rodent nucleus raphe magnus. J Neurosci 2:829-842.

Boivie J, Meyerson BA (1982) A correlative anatomical and clinical study of pain suppression by deep brain stimulation. Pain 13:113-126.

Bushnell MC, Duncan GH, Dubner R, Jones RL, Maixner W (1985) Attentional influences on noxious and innocuous cutaneous heat detection in humans and monkeys. J Neurosci 5:1103-1110.

Duncan GH, Bushnell MC, Bates R, Dubner R (1987) Task-related responses of monkey medullary dorsal horn neurons. J Neurophysiol 57:289-310.

Fields HL, Basbaum AI (1999) Central nervous system mechanisms of pain modulation. In: Textbook of pain (Wall PD, Melzack R, eds), pp 309-329. London: Churchill Livingstone.

Gati JS, Menon RS, Ugurbil K, Rutt BK (1997) Experimental determination of the BOLD field strength dependence in vessels and tissue. Magn Reson Med 38:296-302.

Gaughan AM, Gracely RH (1989) A somatization model of repressed negative emotion: defensiveness increases affective ratings of thermal pain sensations. Soc Behav Med [Abstr].

Good M, Stanton HM, Grass JA, Cranston AG, Choi C, Schoolmeesters LJ, Salman A (1999) Relief of postoperative pain with jaw relaxation, music and their combination. Pain 81:163-172.

Gray TS, Magnuson DJ (1992) Peptide immunoreactive neurons in the amygdala and the bed nucleus of the stria terminalis project to the midbrain central gray in the rat. Peptides 13:451-460.

Hagbarth KE, Kerr DIB (1954) Central influences on spinal afferent conduction. J Neurophysiol 17:295-307.

Hardy SG, Leichnetz GR (1981) Cortical projections to the periaqueductal gray in the monkey: a retrograde and orthograde horseradish peroxidase study. Neurosci Lett 22:97-101.

Head H, Holmes G (1911) Sensory disturbances from cerebral lesions. Brain 34:102-254.

Herbert H, Saper CB (1992) Organization of medullary adrenergic and noradrenergic projections to the periaqueductal gray matter in the rat. J Comp Neurol 315:34-52.

Lavernhe-Lemaire MC, Robier A (1997) Is the afferent auditory message modulated by the cortex (in French)? Arch Physiol Biochem 105:645-654.
Mayer DJ, Price DD (1976) Central nervous system mechanisms of analgesia. Pain 2:379-404.

Mayer DJ, Wolfle TL, Akil H, Carder B, Liebeskind JC (1971) Analgesia from electrical stimulation in the brainstem of the rat. Science 174:1351-1354.

McGlone F, Francis S, Lloyd DM, Newton J, Bowtell R (1999) The role of the superior colliculus in tactile attention: an fMRI study. J Cognit Neurosci [Suppl] 11:56.

Melzack R, Wall PD, Ty TC (1982) Acute pain in an emergency clinic: latency of onset and descriptor patterns related to different injuries. Pain 14:33-43.

Miron D, Duncan GH, Bushnell MC (1989) Effects of attention on the intensity and unpleasantness of thermal pain. Pain 39:345-352.

Moret V, Forster A, Laverriere MC, Lambert H, Gaillard RC, Bourgeois P, Haynal A, Gemperle M, Buchser E (1991) Mechanism of analgesia induced by hypnosis and acupuncture: is there a difference? Pain 45:135-140.

Oliveras J-L, Martin G, Montagne J, Vos B (1990) Single unit activity at ventromedial medulla level in the awake, freely moving rat: effects of noxious heat and light tactile stimuli onto convergent neurons. Brain Res 506:19-30.

Petrovic P, Petersson KM, Ghatan PH, Stone ES, Ingvar M (2000) Pain-related cerebral activation is altered by a distracting cognitive task. Pain 85:19-30.

Ploghaus A, Tracey I, Gati JS, Clare S, Menon RS, Matthews P, Rawlins J (1999) Dissociating pain from its anticipation in the human brain. Science 284:1979-1981.

Ploghaus A, Tracey I, Clare S, Gati JS, Rawlins J, Matthews P (2000) The neural substrate of the prediction error for aversive events. Proc Natl Acad Sci USA 97:9281-9286.

Ploner M, Freund HJ, Schnitzler A (1999) Pain affect without pain sensation in a patient with a postcentral lesion. Pain 81:211-213.

Rainville P, Duncan GH, Price DD, Carrier B, Bushnell MC (1997) Pain affect encoded in human anterior cingulate but not somatosensory cortex. Science 277:968-971.

Reynolds DV (1969) Surgery in the rat during electrical analgesia induced by focal brain stimulation. Science 164:444-445.

Tracey I, Becerra L, Chang I, Breiter H, Jenkins L, Borsook D, Gonzalez RG (2000) Noxious hot and cold stimulation produce common patterns of brain activation in humans: a functional magnetic resonance imaging study. Neurosci Lett 288:159-162.

Wall PD, Melzack R (1999) In: Textbook of pain. London: Churchill Livingstone.

Watkins LR, Mayer DJ (1982) Organization of endogenous opiate and nonopiate pain control systems. Science 216:1185-1192.

Watkins LR, Cobelli DA, Mayer DJ (1982) Classical conditioning of front paw and hind paw footshock induced analgesia (FSIA): naloxone reversibility and descending pathways. Brain Res 243:119-132. 